\title{
Avaliação da posição do supercílio por medidas angulares
}

\author{
Evaluation of eyebrow position using angular measures
}

\author{
Olívia Matai ${ }^{1}$ \\ Marcelo Mendes Lavezzo ${ }^{2}$ \\ Silvana Artioli Schellini ${ }^{3}$ \\ Carlos Roberto Pereira Padovani ${ }^{4}$ \\ Carlos Roberto Padovani ${ }^{5}$
}

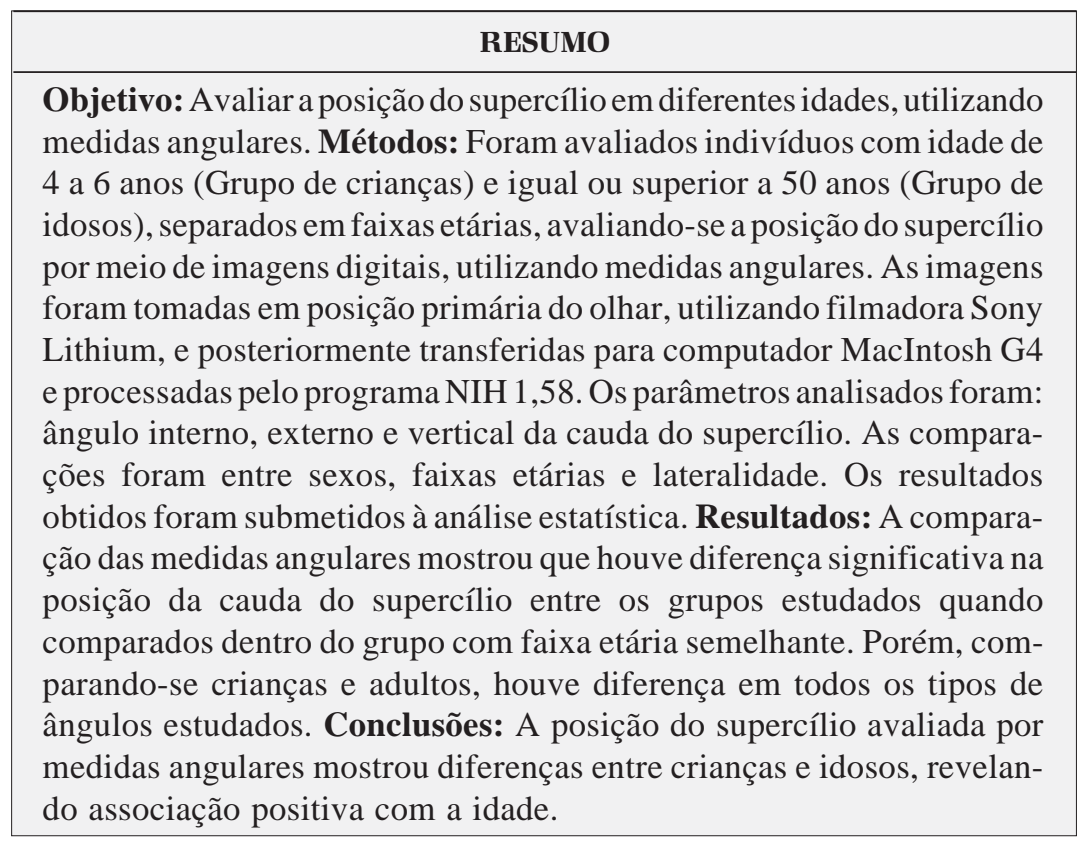

Descritores: Sobrancelhas/anatomia \& histologia; Pálpebras/anatomia \& histologia; Valores de referência; Processamento de imagem assistida por computador/métodos; Criança; Préescolar; Adulto
Trabalho realizado na Faculdade de Medicina da Universidade Estadual Paulista "Júlio Mesquita Filho" UNESP - Botucatu (SP) - Brasil.

${ }^{1}$ Residente do Departamento de Oftalmologia, Otorrinolaringologia e Cirurgia de Cabeça e Pescoço da Faculdade de Medicina da Universidade Estadual Paulista "Júlio Mesquita Filho" - UNESP - Botucatu (SP) - Brasil. ${ }^{2}$ Bolsista de PIBIC do Departamento de Cirurgia da Faculdade de Medicina da UNESP - Botucatu (SP) - Brasil.

${ }^{3}$ Livre-Docente do Departamento de Oftalmologia, Otorrinolaringologia e Cirurgia de Cabeça e Pescoço da Faculdade de Medicina da UNESP - Botucatu (SP) - Brasil.

${ }^{4}$ Pós-Graduando do Departamento de Bioestatística, Instituto de Biociências da Faculdade de Medicina da UNESP - Botucatu (SP) - Brasil.

${ }^{5}$ Professor Titular do Departamento de Bioestatística, Instituto de Biociências da Faculdade de Medicina da UNESP - Botucatu (SP) - Brasil.

Endereço para correspondência: Silvana Artioli Schellini. Departamento de Oftalmologia, Otorrinolaringologia e Cirurgia de Cabeça e Pescoço da Faculdade de Medicina da Universidade Estadual Paulista "Júlio Mesquita Filho" - UNESP - Botucatu (SP) CEP 18618-000 E-mail: sartioli@fmb.unesp.br

Recebido para publicação em 01.03.2006 Aprovação em 30.08.2006

Nota Editorial: Depois de concluída a análise do artigo sob sigilo editorial e com a anuência do Dr. Eurípedes da Mota Moura sobre a divulgação de seu nome como revisor, agradecemos sua participação neste processo.

\section{INTRODUÇÃO}

A posição do supercílio é importante fator determinante da aparência facial $^{(1)}$, principalmente no que diz respeito à sua relação anatômica com as pálpebras $^{(2)}$, sendo de extrema importância nas blefaroplastias e outros procedimentos estéticos ${ }^{(3-4)}$. Entretanto, poucos são os estudos que envolvem esta região ${ }^{(2,5)}$.

A mensuração da excursão normal do músculo frontal mostra que a mobilidade do supercílio é maior em sua porção lateral ${ }^{(5)}$. Porém, a avaliação quantitativa da queda do supercílio relacionada com a idade, usando medidas lineares mostrou que não há variação significativa da posição do supercílio com o envelhecimento ${ }^{(6)}$, já que os pontos tomados como referência para a realização das medidas lineares não abrangiam exatamente a porção do supercílio que teoricamente mais muda, a porção lateral. Desta forma, as medidas lineares deixariam de avaliar o descenso da cauda do supercílio.

Assim, baseados na suposição de que existe queda da cauda do supercílio com o aumento da idade, nos propusemos a realizar esta avaliação utilizando medidas angulares.

Portanto, o objetivo do presente estudo foi conhecer o comportamento do posicionamento do supercílio relacionado com a idade, usando medidas angulares. 


\section{MÉTODOS}

Foram avaliados 261 indivíduos (522 supercílios), sendo 125 crianças, com idade entre 4 a 6 anos (Grupo das crianças), alunos do Colégio La Salle de Botucatu; e 136 indivíduos maiores que 50 anos (Grupo de idosos), pacientes do Hospital das Clínicas da Faculdade de Medicina de Botucatu - UNESP.

Como critério de inclusão para participação do estudo adotou-se que os participantes não deveriam apresentar doença ou cirurgia óculo-palpebral prévia.

As imagens foram tomadas no plano frontal e em posição primária do olhar, utilizando uma filmadora digital Sony Lithium, gravadas em fita cassete, durante pelo menos 3 minutos, sendo posteriormente transferidas para um computador MacIntosh G4 e processadas pelo programa NIH 1.58.

Foram analisados os seguintes parâmetros (Figura 1):

1) Ângulo interno da cauda do supercílio: obtido por meio da intersecção da reta que une o canto medial e lateral, com outra ligando a cauda do supercílio ao canto medial.

2) Ângulo externo da cauda do supercílio: intersecção da reta que une o canto medial e lateral com a linha que liga a cauda do supercílio ao canto lateral.

3) Ângulo vertical da cauda do supercílio: intersecção de
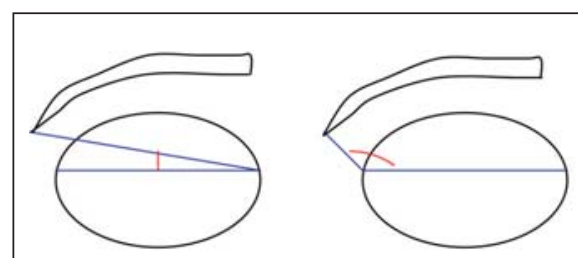

Ângulo 2

Ângulo 1

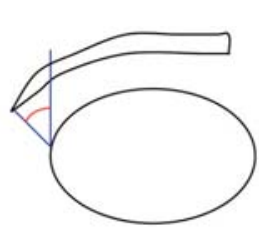

Ângulo 3
Figura 1 - Demonstração dos ângulos medidos para o estudo da posição da cauda do supercílio linha vertical traçada a partir do canto lateral, com reta unindo a cauda do supercílio e o canto externo.

As medidas obtidas foram comparadas nos dois sexos, entre idades (Grupo das crianças) dividido em 4, 5 e 6 anos e (Grupo dos idosos) divididos em faixas etárias de 50 até 60 anos, 61 até 70 anos e maiores que 70 anos de idade e de acordo com a lateralidade (lado direito comparado com esquerdo).

O teste estatístico utilizado foi o teste T de Student para amostras independentes e a técnica da Análise de Variância para o modelo com dois fatores, complementada com o teste de comparações múltiplas de Tukey.

\section{RESULTADOS}

Avaliando-se a média e o desvio padrão das medidas angulares obtidas para o grupo das crianças, observaram-se valores semelhantes para ambos os sexos em todas as idades avaliadas, tanto no olho direito, como no esquerdo. A comparação das crianças das diversas idades mostrou que houve diferença estatística no ângulo 3 do olho direito de meninos de 5 e 6 anos que apresentaram medidas maiores que os de 4 e também no ângulo 3 do olho esquerdo de meninas de 5, que tiveram valores menores que as de 6 anos (Tabela 1).

A avaliação das medidas angulares obtidas no grupo de idosos mostrou que todos os ângulos estudados tiveram resultados semelhantes para ambos os sexos, exceto na faixa etária de maiores de 70 anos, quando houve diferença significativa em todos os ângulos, tanto do lado direito quanto do esquerdo. Porém, a diferença foi variável, sendo o ângulo 1 de valores superiores em mulheres e o 2 e 3 , em homens, tanto para o olho direito como para o olho esquerdo (Tabela 2).

A avaliação comparativa dos dois grupos avaliados, ou seja, a comparação das medidas angulares do grupo das crianças comparadas com o grupo dos idosos, mostrou que houve diferença significativa em todos os ângulos estudados, tanto do lado direito quanto do lado esquerdo (Tabela 3).

\begin{tabular}{|c|c|c|c|c|c|}
\hline \multirow[t]{2}{*}{ Olho } & \multirow[t]{2}{*}{ Ângulo } & \multirow[t]{2}{*}{ Sexo } & \multicolumn{3}{|c|}{ Faixa etária } \\
\hline & & & 4 & 5 & 6 \\
\hline \multirow[t]{6}{*}{ Direito } & \multirow[t]{2}{*}{1} & Masculino & $17,64 \pm 3,76 \mathrm{aA}$ & $16,55 \pm 2,37 \mathrm{aA}$ & $16,28 \pm 3,52 \mathrm{aA}$ \\
\hline & & Feminino & $16,61 \pm 3,28 \mathrm{aA}$ & $15,33 \pm 2,48 a A$ & $16,91 \pm 3,62 \mathrm{aA}$ \\
\hline & \multirow[t]{2}{*}{2} & Masculino & $136,40 \pm 7,14 \mathrm{aA}$ & $139,75 \pm 8,60 \mathrm{aA}$ & $140,71 \pm 8,72 \mathrm{aA}$ \\
\hline & & Feminino & $139,17 \pm 11,03 a A$ & $139,69 \pm 6,04 \mathrm{aA}$ & $138,42 \pm 9,44 \mathrm{aA}$ \\
\hline & \multirow[t]{2}{*}{3} & Masculino & $38,89 \pm 7,58 \mathrm{aA}$ & $46,48 \pm 7,80 \mathrm{aB}$ & $44,82 \pm 8,50 \mathrm{aB}$ \\
\hline & & Feminino & $41,89 \pm 7,37 \mathrm{aA}$ & $43,83 \pm 8,08 \mathrm{aA}$ & $42,52 \pm 6,72 \mathrm{aA}$ \\
\hline \multirow[t]{6}{*}{ Esquerdo } & \multirow[t]{2}{*}{1} & Masculino & $18,68 \pm 3,56 a A$ & $18,47 \pm 3,16 a A$ & $18,90 \pm 6,75 \mathrm{aA}$ \\
\hline & & Feminino & $16,98 \pm 3,34 \mathrm{aA}$ & $18,12 \pm 3,44 \mathrm{aA}$ & $18,55 \pm 2,75 \mathrm{aA}$ \\
\hline & \multirow[t]{2}{*}{2} & Masculino & $132,81 \pm 8,65 \mathrm{aA}$ & $133,25 \pm 16,58 \mathrm{aA}$ & $135,24 \pm 8,89 a A$ \\
\hline & & Feminino & $135,40 \pm 9,97 \mathrm{aA}$ & $129,89 \pm 13,64 a A$ & $135,48 \pm 7,10 \mathrm{aA}$ \\
\hline & \multirow[t]{2}{*}{3} & Masculino & $39,26 \pm 9,10 \mathrm{aA}$ & $38,49 \pm 7,54 \mathrm{~A}$ & $41,56 \pm 7,71 \mathrm{aA}$ \\
\hline & & Feminino & $37,33 \pm 8,87 \mathrm{aAB}$ & $33,86 \pm 7,56 \mathrm{~A}$ & $40,52 \pm 6,76 \mathrm{aB}$ \\
\hline
\end{tabular}




\begin{tabular}{|c|c|c|c|c|c|}
\hline \multirow[t]{2}{*}{ Olho } & \multirow[t]{2}{*}{ Ângulo } & \multirow[t]{2}{*}{ Sexo } & \multicolumn{3}{|c|}{ Faixa etária } \\
\hline & & & $50-60$ & $61-70$ & $>70$ \\
\hline \multirow[t]{6}{*}{ Direito } & 1 & Masculino & $11,05 \pm 5,15 \mathrm{aA}$ & $9,65 \pm 5,46 \mathrm{aA}$ & $8,31 \pm 6,87 \mathrm{aA}$ \\
\hline & & Feminino & $13,68 \pm 5,63 a A$ & $10,61 \pm 4,49 a A$ & $12,86 \pm 6,56 \mathrm{bA}$ \\
\hline & 2 & Masculino & $147,09 \pm 13,91 \mathrm{aA}$ & $152,15 \pm 15,45 \mathrm{aA}$ & $156,65 \pm 18,24 b A$ \\
\hline & & Feminino & $142,20 \pm 13,99 a A$ & $148,19 \pm 12,45 a \mathrm{~A}$ & $145,92 \pm 17,06$ aA \\
\hline & 3 & Masculino & $53,23 \pm 13,95 \mathrm{aA}$ & $56,19 \pm 13,01 \mathrm{aA}$ & $64,27 \pm 20,92 \mathrm{bA}$ \\
\hline & & Feminino & $48,18 \pm 14,44 a A$ & $54,07 \pm 12,43 a A$ & $50,49 \pm 18,53 a A$ \\
\hline \multirow[t]{6}{*}{ Esquerdo } & 1 & Masculino & $10,00 \pm 5,77 \mathrm{aA}$ & $10,51 \pm 6,74 \mathrm{aA}$ & $8,52 \pm 7,70 \mathrm{aA}$ \\
\hline & & Feminino & $13,41 \pm 5,85 \mathrm{aA}$ & $10,57 \pm 4,65 \mathrm{aA}$ & $12,66 \pm 7,89 \mathrm{bA}$ \\
\hline & 2 & Masculino & $150,50 \pm 16,23 \mathrm{aA}$ & $148,46 \pm 18,05 a A$ & $157,20 \pm 19,23 b A$ \\
\hline & & Feminino & $143,11 \pm 13,76$ aA & $149,30 \pm 10,87 \mathrm{aA}$ & $144,32 \pm 20,41 a A$ \\
\hline & 3 & Masculino & $58,35 \pm 11,30 \mathrm{aA}$ & $54,27 \pm 16,88 \mathrm{aA}$ & $66,14 \pm 19,34 \mathrm{bA}$ \\
\hline & & Feminino & $51,19 \pm 14,03 \mathrm{aA}$ & $56,58 \pm 12,88 a A$ & $55,62 \pm 23,21 a A$ \\
\hline
\end{tabular}

\begin{tabular}{|c|c|c|c|c|}
\hline \multirow[t]{2}{*}{ Olho } & \multirow[t]{2}{*}{ Ângulo } & \multicolumn{2}{|c|}{ Grupo } & \multirow{2}{*}{$\begin{array}{l}\text { Resultado do } \\
\text { teste estatístico }\end{array}$} \\
\hline & & Idosos & Crianças & \\
\hline \multirow[t]{3}{*}{ Direito } & 1 & $11,16 \pm 5,58$ & $16,59 \pm 3,28$ & $P<0,0001$ \\
\hline & 2 & $148,15 \pm 14,88$ & $139,21 \pm 9,10$ & $P<0,0001$ \\
\hline & 3 & $53,76 \pm 15,18$ & $43,24 \pm 7,69$ & $P<0,0001$ \\
\hline \multirow[t]{3}{*}{ Esquerdo } & 1 & $11,06 \pm 6,31$ & $18,23 \pm 4,30$ & $P<0,0001$ \\
\hline & 2 & $148,36 \pm 16,16$ & $134,30 \pm 10,86$ & $P<0,0001$ \\
\hline & 3 & $55,81 \pm 16,18$ & $39,02 \pm 7,96$ & $P<0,0001$ \\
\hline \multirow[t]{2}{*}{ Ambos } & $\begin{array}{l}1 \\
2\end{array}$ & $\begin{array}{r}11,11 \pm 5,94 \\
148,25 \pm 15,50\end{array}$ & 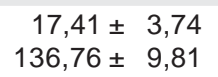 & $\begin{array}{l}P<0,0001 \\
P<0,0001\end{array}$ \\
\hline & 3 & $54,78 \pm 15,62$ & $41,13 \pm 7,81$ & $P<0,0001$ \\
\hline
\end{tabular}

\section{DISCUSS ÃO}

A posição do supercílio e das pálpebras tem importância na expressão facial e está relacionada com a emoção. Assim, mudanças, mesmo que mínimas, alteram a expressão facial ${ }^{(7)}$.

Alguns fatores podem ter influência na posição do supercílio, tais como a gravidade, o sexo, a idade e a raça. Apesar de conhecer que o supercílio sofre influência racial, o que foi demonstrado por avaliação feita na Malásia e que estabeleceu os valores para aquela população ${ }^{(8)}$, no presente estudo não se levou em conta este fator, uma vez que a maioria da população brasileira pode ser considerada mestiça.

O sexo e a idade podem ter influência na posição do supercílio $^{(9)}$. Mulheres possuem o supercílio cerca de $2,5 \mathrm{~mm}$ mais alto que os homens ${ }^{(10)}$. Porém, este dado não se confirma estatisticamente nas avaliações por medidas angulares, apesar da tendência de valores maiores de ângulo 1 e menores de ângulo 2 no sexo feminino.

Outros estudos confirmam que, com o aumento da idade, ocorre variação da posição da pálpebra e do supercílio, com altos valores de correlação ${ }^{(2)}$, sendo o envelhecimento primeiramente visível na região têmporo-orbital, quando a ptose e o "pseudo-excesso" de pele na pálpebra superior ocorrem também por queda da cauda do supercílio ${ }^{(11)}$.
Sabendo-se que a porção lateral do supercílio é a primeira a apresentar ptose, medidas angulares da cauda do supercílio foram feitas neste estudo, a fim de melhor refletir a situação desta posição, uma vez que medidas lineares falham em demonstrar esta alteração posicional ${ }^{(6)}$, já que o supercílio tem a forma de uma parábola. Também fica difícil utilizando medidas lineares que se tenha um ponto de referência no local da cauda do supercílio.

No presente estudo, a presença do ângulo 1 mais agudo, ângulo 2 mais obtuso e ângulo 3 maior em idosos, demonstraram a queda da cauda do supercílio dos idosos, quando comparados idosos e crianças.

Estudos anatômicos foram feitos com o intuito de melhor entender a patogênese da ptose do supercílio e o porquê da porção lateral ser a primeira a apresentar-se com ptose. Verificou-se que existe um coxim gorduroso abaixo do supercílio que atinge até a porção mediana do mesmo e que densas ligações existem entre o supercílio e esta gordura, mantendo o mesmo acima da rima orbitária. A parte lateral não possui estas adesões, sendo a porção que fica mais frouxamente aderida por se dar, neste local, as linhas de expressão facial ${ }^{(12-13)}$.

$\mathrm{Na}$ porção lateral do supercílio existe um ligamento que resulta da coalescência das fáscias temporal e da gálea, resul- 
tando num espesso ligamento que sustenta a cauda do supercílio. Nos idosos, a maior mobilidade do supercílio lateral deve ser resultante da frouxidão deste ligamento ${ }^{(5)}$. E esta maior mobilidade deve estar relacionada com o descenso desta região.

\section{CONCLUSÃO}

Existem diferenças significativas entre a posição do supercílio de crianças e idosos. Medidas angulares são capazes de demonstrar estas diferenças.

\section{ABSTRACT}

Purpose: To evaluate the eyebrow position in children and elderly using angle measurements. Methods: The eyebrow position was evaluated by digital images in 4 to 6 -year-old children (children group) and in patients with 50 years or more (elderly group), separated by age range. The images were taken in the primary gaze position with a Sony Lithium camera and then transferred to a MacIntosh G4 computer and processed by the NIH 1.58 program. The eyebrow position was evaluated using angle measures. Comparisons were done according to age, sex and laterality and the data were submitted to statistical analysis. Results: The comparison of the angular measures showed that there was a significant difference in the position of eyebrow position between the studied groups with the same age. But comparing children and elderly there were differences in all performed measurements. Conclusion: The eyebrow external angle positions showed differences bet- ween children and elderly with a positive association between eyebrow position and age.

Keywords: Eyebrows/anatomy \& histology; Eyelids/anatomy \& histology; Reference values; Image processing, computerassisted/methods; Child; Child, pre-school; Adult

\section{REFERÊNCIAS}

1. Ergun SS, Sahinoglu K. Eyebrow transplantation. Ann Plast Surg. 2003;51 (6):584-6.

2. Cartwright MJ, Kurumety UR, Nelson CC, Frueh BR, Musch DC. Measurements of upper eyelid and eyebrow dimensions in healthy white individuals. Am J Ophthalmol. 1994;117(2):231-4.

3. Oestreicher JH, Hurwitz JJ. The position of the eyebrow. Ophthalmic Surg. 1990;21(4):245-9.

4. Koch RJ, Pope K. Quantitative assessment of brow position: a new measurement system. Plast Reconstr Surg. 2004;113(4):1290-1.

5. Cruz JRS. Quantificação da ação do músculo frontal através da medida da excursão do supercílio [tese]. Ribeirão Preto: Faculdade de Medicina de Ribeirão Preto da Universidade de São Paulo; 2003. 43p.

6. Siqueira M, Joaquim A, Schellini SA, Padovani CR, Cruz AAV. Alterações palpebrais após a idade de 50 anos. Arq Bras Oftalmol. 2005;68(3):285-90.

7. Karabulut AB, Tumerdem B. Forehead lift: a combined approach using subperiosteal and subgaleal dissection planes. Aesthetic Plast Surg. 2001;25(5):378-81

8. Dharap AS, Reddy SC. Upper eyelid and eyebrow dimensions in Malays. Med J Malaysia. 1995;50(4):377-81.

9. Niechajev I. Transpalpebral browpexy. Plast Reconstr Surg. 2004;113(7): 2172-80; discussion 2181.

10. Van den Bosch WA, Leenders I, Mulder P. Topographic anatomy of the eyelids, and the effects of sex and age. Br J Ophthalmol. 1999;83(3):347-52.

11. Leopizzi G. A transpalpebral approach to treatment of eyebrow ptosis. Aesthetic Plast Surg. 1999;23(2):125-30.

12. Lemke BN, Stasior OG. The anatomy of eyebrow ptosis. Arch Ophtalmol. 1982;100(6):981-6.

13. Knize DM. An anatomically based study mechanism of eyebrow ptosis. Plast Reconstr Surg. 1996;97(7):1321-33.

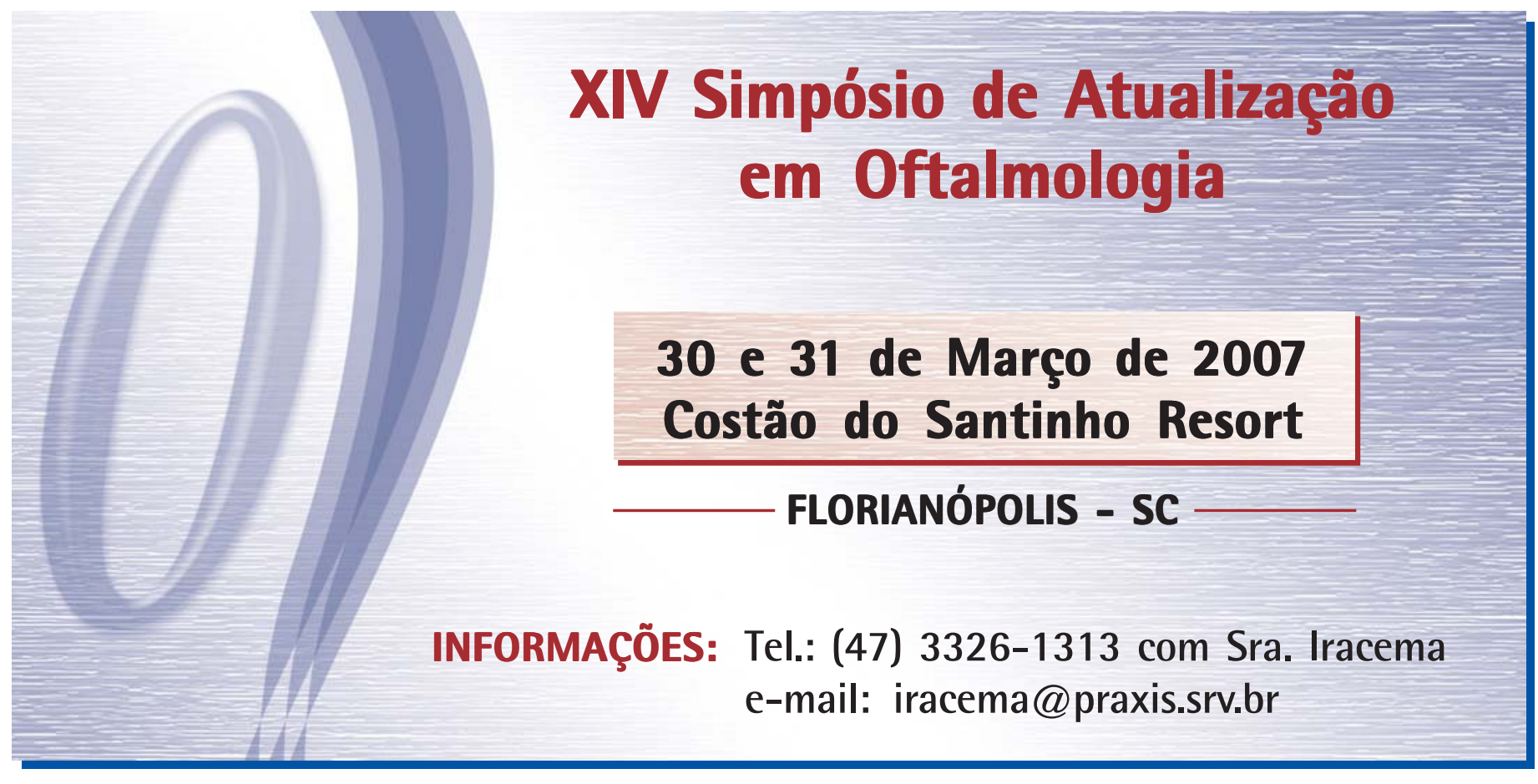

